\title{
O que te Apetece Mais? O Impacto da Imagem na Percepção de Sabor no Consumo de Alimentos Não Saudáveis
}

\author{
What pleases you the most? The impact of food image on perception of taste in \\ the consumption of non-healthy food
}

\author{
Francisco Eduardo Aoyama Nogueira ${ }^{1}$ \\ Rodolfo Coral Azambuja ${ }^{2}$ \\ Juliana Martins Diefenthäler ${ }^{3}$ \\ Carolina Barth ${ }^{4}$
}

\begin{abstract}
Resumo
A influência de outros indivíduos na percepção de sabor pelo consumidor para alimentos saudáveis e não saudáveis é tema recente na literatura de marketing (POOR et al., 2013). Resultados evidenciam que, para alimentos considerados não saudáveis, há um efeito positivo quando o ato de comer está destacado em uma imagem apresentada ao consumidor previamente ao seu próprio consumo. Este artigo objetiva avançar nos estudos relacionados com a influência de outros indivíduos na percepção de sabor, considerando o impacto do meio de apresentação do estímulo - com a presença de outro consumindo o alimento, fisicamente no mesmo ambiente ou em imagens impressas-e as variações no tipo físico e no gênero dessa outra pessoa. Foi realizado um experimento com 180 estudantes, com design between-subjects factorial, no formato 2 (forma de apresentação do estímulo: impresso ou presencial) x 2 (tipo físico do endossante: magro ou acima do peso) $\times 2$ (gênero do endossante: feminino ou masculino). Os resultados evidenciam que o tipo físico da pessoa consumindo o alimento influencia a percepção de sabor pelo consumidor. Em uma interação com o gênero, este estudo mostra que a exposição a um indivíduo do sexo masculino acima do peso resulta em uma percepção de sabor menor do alimento, quando comparado a indivíduos magros do sexo masculino ou do sexo feminino, independentemente do tipo físico. Desse modo, o presente estudo contribui para a teoria de percepção de sabor, ampliando os resultados encontrados por Poor et al. (2013), bem como para os estudos relacionados à propaganda.
\end{abstract}

Palavras-chave: Percepção de sabor. Alimentos não saudáveis. Design experimental.

\begin{abstract}
The influence of other people in the perception of food taste for both healthy and non-healthy food is a recent topic in the Marketing literature (Poor et al., 2013). The results show that for non-healthy food, there is a positive effect when the act of eating is shown in the image to the consumer right before consumption. The objective of this article is to extend studies related to the influence of other people in the perception of food's taste, in regards to the stimulus shown (i.e. in the presence of someone else - the endorser - physically present eating the food or through the presence of a printed image), on the same physical built and gender. It was conducted a between-subjects factorial design 2 (physical presence vs. Printed) x 2 (endorser physical built: thin vs fat) with 180 students. The results show that the endorser's physical built eating the food will influence the perception of taste for food. An interaction was found between gender demonstrating that for fat male endorsers the perception of taste is lesser than it is for thin male endorsers or female endorsers of both fat and thin built. Thus, the present study contributes to the theory of perception of taste, extending results found by Poor et al., (2013), as well as for studies relating to food advertising.
\end{abstract}

Keywords: Perception of taste. Non-healthy food. Experimental design.

Doutorado em andamento pela Universidade Federal do Rio Grande do Sul (EA/UFRGS). Período de doutorado sanduíche na HÉC de Montréal no Canadá. Brasil. Afiliação: UFRGS. Lattes: http://lattes.cnpq.br/2602207100895710 Email: franciscoeanogueira@yahoo.com.br

2 Mestrado em andamento em Administração pela Universidade Federal do Rio Grande do Sul, UFRGS. Brasil. Afiliação: UFRGS. Lattes: http:// lattes.cnpq.br/3691118271447824 Email: rodolfoazambuja@gmail.com

Mestre em Administração pela Universidade Federal do Rio Grande do Sul - UFRGS. Brasil. Lattes: http://lattes.cnpq.br/7177481191509426 Email: juuuh90@hotmail.com

4 Mestre em Administração na área de Marketing pela Universidade Federal do Rio Grande do Sul. Brasil. Afiliação: UFRGS. Lattes: http://lattes. cnpq.br/5221030118959521 Email: carolina_barth@yahoo.com.br 


\section{Introdução}

Pesquisa realizada por Poor et al. (2013) analisou a influência de outros consumidores na percepção de sabor no consumo de alimentos saudáveis e não saudáveis e evidenciou que, para alimentos conhecidos como não saudáveis (fast foods etc), há um efeito positivo quando o ato de comer está consumado em uma imagem apresentada ao consumidor. $O$ trabalho dos autores mostra, ainda, que há poucos estudos a respeito de quando, como e em quais circunstâncias a exposição a determinados tipos de imagens influencia as respostas dos consumidores em relação às percepções de sabor acerca de determinado produto (POOR et al., 2013).

Em uma situação de grupo, a escolha de um indivíduo pode afetar a dos demais presentes de acordo os objetivos de cada um naquele momento, que são dependentes de aspectos psicológicos e sociais (ARIELY; LEVAV, 2000). Esses objetivos podem fazer o indivíduo convergir ou divergir em relação a uma escolha de grupo. A presença de outras pessoas em uma situação de consumo pode, dessa forma, alterar a decisão que o indivíduo tomaria em relação às opções disponíveis no cardápio de um restaurante ou de uma cervejaria, por exemplo. Imagine que você está indo pela primeira vez em um restaurante com um grupo de amigos. No momento de efetuar os pedidos, um deles que já foi àquele local anteriormente, pede um item do cardápio. Dada essa decisão, os demais integrantes do grupo, com maior probabilidade, escolherão outro item do cardápio ou haverá convergência nas escolhas? E caso ninguém tenha ido anteriormente, imagine que, na chegada, caminhando até a sua mesa, você e seus amigos veem alguém comendo algo que parece interessante, e alguém pergunta o que é. Nesse caso, como seriam as escolhas posteriores? Qual o papel da expectativa de sabor na escolha? Qual o impacto dessa exposição prévia a alguém consumindo o alimento na avaliação desse mesmo alimento, após o consumo, por parte do consumidor?

Poor et al. (2013) tiveram como objetivo investigar o relacionamento entre a exposição à imagem e a percepção de sabor, concluindo que esse relacionamento depende, primeiramente, do tipo de alimento - para alimentos saudáveis não houve diferenças entre as manipulações realizadas. Em seguida, do tipo de imagem - sendo constatado que a imagem de consumação, do ato de comer, produz efeito positivo na percepção de sabor em relação aos demais tipos de imagem. Desse modo, este estudo buscará analisar, primeiramente, o possível efeito do meio de apresentação da imagem - neste caso, a diferença existente entre uma imagem real (alguém consumindo o alimento no mesmo ambiente, fisicamente) e impressa - na percepção de sabor pelo indivíduo.

O efeito do meio de apresentação da imagem constitui tema de grande relevância, tanto do ponto de vista teórico quanto para o campo gerencial, pois contribui para o constructo de percepção e influencia no que diz respeito à disposição de lojas e restaurantes. Por isso, os autores já sugeriam tal investigação como ponto de partida para futuros estudos relacionados ao tema.

Autores no campo de marketing investigaram aspectos relacionados com as características do perfil do endossante de determinado anúncio (como o tipo físico, etnia e gênero) afetam a avaliação que os consumidores fazem da qualidade do produto e da marca (p.e. YANG; ZHANG; PERACCHIO, 2010; BJERKE; POLEGATO, 2006). Tipos físicos estão ligados à ideia de beleza, sendo a escolha do tipo físico ideal para um anúncio um importante preditor do sucesso da peça.

Contudo permanece a questão do que faz uma imagem - relacionada ao consumo de alimentos não saudáveis - aumentar a percepção de sabor ou o desejo de consumir determinado alimento. Consumidores tendem a buscar justificativas para os seus comportamentos (OKADA, 2005). O tipo físico de outras pessoas, no ato de consumo, consoante evidenciado por McFerran et al. (2010a), pode influenciar a escolha da quantidade a ser consumida de determinado alimento, pois quando a decisão é ancorada no consumo de pessoas obesas, a quantidade escolhida é menor do que quando o parâmetro é uma pessoa magra. Poor et al. (2013) evidenciam que a imagem do consumo de alimentos não saudáveis serve como uma espécie de justificativa psicológica e, desse modo, reduz o conflito interno do consumidor relacionado ao tipo de alimento, aumentando a percepção de sabor.

Embora, separadamente, existam trabalhos sobre ambos os temas, não há uma abordagem prévia acerca de como determinados tipos físicos podem estar relacionados à percepção de sabor no consumo de 
comidas não saudáveis. Assim, o presente artigo busca também investigar se há relação entre o tipo físico do indivíduo presente na imagem relacionada ao consumo de alimentos não saudáveis, além da percepção de sabor que o consumidor estabelece em relação ao produto. Espera-se que haja efeito similar àquele existente na escolha das quantidades consumidas.

O presente estudo pretende avançar na teoria sobre percepção de sabor, testando o impacto do meio de apresentação do estímulo (imagem consumatória), do tipo físico e do gênero do indivíduo presente no estímulo. Para isso, foi desenvolvido um estudo experimental em laboratório, do qual participaram 180 estudantes de graduação em Administração da Universidade Federal do Rio Grande do Sul. Os resultados evidenciam que, embora o meio pelo qual o consumidor é exposto ao estímulo não afete sua percepção de sabor do alimento após o consumo, o tipo físico e a interação com o gênero do endossante impactam em como o indivíduo avalia o alimento não saudável.

\section{Referencial Teórico}

A seguir, será realizada uma revisão teórica sobre percepção de sabor e o papel da imagem nessa percepção. Também serão revistos estudos a respeito da influência do tipo físico do endossante presente na imagem, bem como a influência do meio de apresentação nessa relação, seja uma imagem impressa, seja a presença física no local de consumo.

\section{Percepção de Sabor}

Atualmente, é reconhecido que o olfato é um dos sentidos mais importantes vinculados ao paladar e à percepção de sabor. Entretanto todos os demais sentidos exercem importante influência nesse mesmo constructo, sendo a visão um dos fatores essenciais. A visão atua como um dos sentidos primários em relação aos alimentos, criando expectativas e percepções a respeito do sabor e do valor nutricional das opções disponíveis que dirigem a aceitação ou rejeição de determinado produto (OHLA et al., 2012). Os autores afirmam, ainda, que a percepção de sabor se constitui em um dos principais elementos para a avaliação do consumidor. Os elementos visuais, desse modo, constituem importante ferramenta nas mãos do profissional de marketing interessado em estabelecer conexões com seu públicoalvo.

A percepção de sabor é influenciada por muitas outras sensações, por exemplo, textura, temperatura e cheiro da comida, mas também pelas expectativas com base na experiência e memória associada a um dado alimento ou sabor anterior (OHLA et al., 2010). O sabor é talvez a mais multimodal entre todas as nossas experiências sensoriais (SMALL, 2012). Mizutani et al. (2012) afirmam que a lembrança de sabor de determinado alimento pode ser tão importante como o sabor percebido em si, principalmente em situações em que a seleção e compra de produtos não dispõem de degustação ou teste, de modo que o consumidor precisa confiar em suas experiências anteriores. Entretanto, as memórias armazenadas nem sempre são exatas; nesse caso, os estímulos recebidos durante a memorização e recordação podem influenciar a percepção armazenada.

Um alimento é identificado a partir de seu sabor e constitui-se de um fator determinante para a aceitação do consumidor (DATTATREYA et al., 2002). Tendo em vista que o sabor é considerado um dos atributos mais importantes que determinam a aceitação dos alimentos pelo consumidor (GUICHARD, 2002), é evidente a necessidade de estudo e exploração da percepção do sabor em diversos campos da ciência, procurando estabelecer ligações entre os constructos vinculados ao consumidor.

O desenvolvimento de produtos na indústria de alimentos, envolve, muitas vezes, testes comparativos (GIVON; GOLDMAN, 1987). Outros estudos também exploram a percepção de sabor por meio de tarefas verbais. A tarefa de fluência verbal (FV) é um teste utilizado para examinar a percepção cognitiva (GHEMULET et al., 2014). No estudo, os autores exploram uma possível relação entre percepção de sabor nas categorias de base do paladar (doce, salgado, azedo e amargo) e preferências de gosto das pessoas, utilizando uma tarefa de FV em indivíduos saudáveis e disfásicos, além de correlacionar a tarefa de FV com o índice de massa corporal (IMC) dos indivíduos, sugerindo que índices mais elevados se correlacionam com mais respostas em algumas ou todas as categorias e indicam preferência por sabores doces. Mizutani et al. 
(2010), todavia, ressalvam que, embora a influência da informação textual na avaliação do sabor tenha sido bem estudada, a influência de imagens permanece inexplorada.

Apesar de recentemente explorado, o estudo sobre percepção de sabor na pesquisa do consumidor possui diversas aplicações e campos de estudo, sobretudo envolvendo experimentos práticos e explorando novas abordagens. Os consumidores podem frequentemente provar um alimento antes de comprá-lo. Isto acontece por meio de eventos iniciados pelo comerciante, como degustação de vinhos (p.e. LYNCH; ARIELY, 2000), ou de oportunidades para saborear um item que outra pessoa, como um membro da família ou amigo, está comendo (SHIV; NOWLIS, 2004). Esses autores conduzem uma pesquisa relacionando o fator da distração vinculado à degustação de alimentos, demonstrando que a distração aumenta a escolha subsequente da degustação da amostra. Além disso, sugerem, ainda, que o consumo é composto por um componente informativo, relacionado aos aspectos informacionais, e um afetivo, ligado a avaliações mais sensoriais, como a percepção de sabor, e que a distração leva a um aumento do impacto do componente afetivo (POOR et al. 2013).

Elder e Krishna (2010) propõem que os anúncios de conteúdo para os produtos alimentares podem afetar a percepção de sabor por meio de cognições sensoriais. Os anúncios multissensoriais resultam em percepções de sabor mais elevadas do que os anúncios com foco em sabores isolados (ELDER; KRISHNA, 2010). Os estudos conduzidos por Ohla et al. (2010) sugerem que a informação visual afeta o processamento de estímulos de gosto subsequentes, em particular a sua avaliação hedônica. Além disso, em artigo posterior, Ohla et al. (2012) esclarecem que olhar para imagens de alimentos produz atividade cerebral que afeta a percepção de sabor. Isto não é surpreendente, visto que elementos visuais constituem um input sensorial primário, indicando a disponibilidade e palatabilidade dos alimentos em estágio pré-ingestivo (OHLA et al., 2012).

Mizutani et al. (2012) relataram que estudos anteriores, focados nos efeitos da informação visual na percepção de sabor, levaram em consideração informações como cor, inscrições textuais e imagens das embalagens. Entretanto pouco ainda há a respeito dos efeitos de diferentes tipos físicos de endossantes ou meios como a imagem se apresenta para o consumidor - se via anúncio ou pelo contato com outras pessoas ingerindo o alimento no mesmo local de consumo. Poor et al. (2013) relatam, ainda, trabalhos relacionados à influência do uso de imagens na formação de atitudes em relação à marca, nas estratégias de processamento de informação, nas respostas emocionais, nas inferências sobre produtos, entre outros.

\section{O Efeito da Imagem na Percepção}

Pode-se facilmente verificar na literatura a existência de extenso número de pesquisas examinando como a apresentação de alimentos pode influenciar as percepções, atitudes e comportamentos (CHERNEV, 2011; MISHRA; CHANDON, 2011). Poor et al. (2013) argumentam, entretanto, que pouco se sabe sobre como a exposição às imagens de comida pode influenciar a percepção de sabor pelo consumidor. Os autores testaram a relação entre exposição a diversos tipos de imagem, relacionadas tanto ao consumo de comidas saudáveis como não saudáveis, e a percepção de sabor. Entretanto, entre as limitações do estudo, identificouse que a análise realizada pelos autores não compreende variações no indivíduo que está consumindo o alimento na imagem ou nos meios de apresentação da imagem aos sujeitos participantes da amostra.

Outros trabalhos evidenciaram que o uso de imagens em marketing tem forte impacto sobre o consumidor, incluindo sobre suas respostas emocionais (CHOWDHURY; OLSEN; PRACEJUS, 2008), sua inferência de produtos (UNDERWOOD; KLEIN, 2002) e sobre seu volume de consumo (MADZHAROV; BLOCK, 2010). Partindo do pressuposto de que a imagem produz efeitos na percepção do consumidor, diversos estudos têm trabalhado também estratégias visuais (ex. complexidade visual, ângulo da câmera, uso de imagens artísticas e não artísticas), com o objetivo de investigar como essas diferentes abordagens podem potencializar os efeitos produzidos na avaliação que o consumidor faz de determinado produto (POOR et al. 2013).

As teorias existentes no âmbito de normas sociais e seus impactos nas escolhas alimentares dos indivíduos evidenciam que o consumo se altera sempre que há a presença de outros consumidores, afetando as escolhas e a quantidade de consumo (McFERRAN et al., 2010a). Killgore et al. (2003) demonstraram que 
a apresentação visual de alimentos de altas calorias, em comparação com a apresentação de alimentos de baixas calorias, produz maior ativação em regiões cerebrais envolvidas em emoção, motivação e antecipação de recompensa.

A conexão entre consumo e percepção de sabor está presente nos estudos entre afeto e cognição no processo decisório (NOWLIS; SHIV, 2005) e também em pesquisas sobre a dor (READ et al., 1999). A imagem de comidas não saudáveis pode induzir o conflito entre o efeito positivo, relacionado ao prazer e gratidão do consumo, e a cognição negativa, que pertence às consequências relacionadas à saúde do consumo (KILLGORE et al., 2003). É uma linha também suportada pela neurociência, que sugere uma relação negativa entre experiência de conflito e experiência de prazer (McCLURE et al., 2007). Em contraste, comidas saudáveis não provocam o mesmo conflito dessa exposição a alimentos.

$\mathrm{O}$ consumidor, quando submetido a imagens de alimentos, tem ativadas áreas no cérebro ligadas à percepção de sabor e estabelece expectativas a respeito do gosto e outros aspectos hedônicos da comida (OHLA et al., 2012). Os autores reportam que imagens relacionadas a alimentos de alto valor calórico aumentam a avaliação hedônica do alimento e, consequentemente, a percepção de sabor - o que não acontece para alimentos saudáveis. Elementos do contexto também se mostraram importantes no processo de avaliação e, dessa forma, devem ser levados em consideração quando se realizam estudos relacionados ao sabor. Além disso, a percepção prévia relacionada ao sabor de alimentos não saudáveis, de acordo com Chandon e Wansink (2012), persistem mesmo após o consumo, corroborando os resultados das investigações de Poor et al. (2013) em relação aos efeitos de diferentes tipos de imagem relacionados a alimentos saudáveis e não saudáveis.

Os autores evidenciam que até mesmo a imagem de um logotipo que represente tal conceito gera expectativas negativas, diminuindo a percepção de sabor relacionada ao alimento (LIEM et al., 2012). Tal efeito é aumentado para aqueles consumidores que possuem opiniões contrárias ao sabor de produtos saudáveis.

\section{Tipo Físico e a Percepção de Sabor}

As variações de tipo físico em imagens e sua influência em percepção de sabor de produtos foram pouco trabalhadas até o momento. Pesquisas anteriores, vinculadas ao tema, estudaram variações no tipo físico de modelos endossantes em anúncios e sua influência na atitude do consumidor em relação à marca. Também foram estudadas variações étnicas nos endossantes (D'ALESSANDRO; CHITTY, 2011) e o tipo ideal de beleza em diferentes contextos culturais (BJERKE; POLEGATO, 2006).

Nesse contexto, é relevante ressaltar que as características particulares de cada consumidor podem desempenhar um papel crítico na forma como os indivíduos percebem propriedades estilísticas de imagens e podem vir a afetar suas avaliações do produto anunciado (YANG; ZHANG; PERACCHIO, 2010). De acordo com a teoria do autoconceito, a autoimagem é a percepção da pessoa em suas próprias habilidades, características, limitações, aparências e personalidade (ROSENBERG, 1979). Os consumidores frequentemente compram produtos que mantêm e melhoram sua autoimagem. As decisões de compra são baseadas no significado simbólico do produto e imagem, que podem ser utilizados para criar e melhorar a autoimagem (LEVY, 1959; SOLOMON; DOUGLAS, 1987). A influência da autoimagem na preferência do consumidor por determinadas marcas é resumida no conceito de congruência da imagem. Vários estudos encontraram uma relação positiva entre a congruência da imagem (entre imagem da marca e a autoimagem) e atitudes para tais produtos (carros, produtos de limpeza, cigarros, revistas, mobília, roupas etc) (HONG; ZINKHAN, 1995).

Os profissionais de marketing devem estar cientes de como a representação dos ideais de corpo e o contexto cultural que se torna associado aos endossantes podem afetar a eficácia das comunicações de marketing (BJERKE; POLEGATO, 2006). Endossantes femininas em publicidade geralmente representam o autoconceito ideal social, com um estereótipo magro (FORBES et al., 2001).

D'Alessandro e Chitty (2011) trabalharam, especificamente, com a influência étnica e o estereótipo feminino físico ideal dos endossantes em anúncios. Os autores também examinaram a efetividade de formatos de corpos mais similares à realidade humana, como os utilizados pela campanha Dove: Beleza Real. Como 
resultado, foi encontrado que a utilização de modelos magras em anúncios provoca o desenvolvimento de uma atitude mais favorável à marca anunciada do que o uso de modelos gordas. Em contrapartida, a utilização de corpos com formatos físicos mais próximos à realidade média da população não apresentou diferenças estatísticas na credibilidade e atratividade do anúncio. Também não foi encontrado nenhum efeito direto da etnia do endossante nessa pesquisa.

Smeesters, Mussweiler e Mandel (2009) também analisaram a forma como anúncios contendo modelos magros ou pesados influenciam a autoestima dos consumidores com excesso de peso, consumidores com peso normal e consumidores com baixo peso. Os resultados apresentaram diferenças para as dimensões comparadas.

Por sua vez, McFerran et al. (2010b) pesquisaram como as escolhas alimentares das pessoas podem ser influenciadas pelo tipo de corpo de quem as está servindo. Enquanto clientes que não estavam de dieta comeram mais quando o atendente era magro, clientes que estavam de dieta comeram mais quando o atendente era mais pesado. Dessa forma, clientes em dieta foram mais persuadidos pelo atendente mais pesado, escolhendo alimentos saudáveis ou não saudáveis quando recomendados pelo atendente. Os resultados foram atribuídos à identificação do cliente com quem o estava atendendo.

Como evidenciado, a influência do tipo físico já foi analisada em relação a diversos constructos de marketing. Entretanto, no contexto de alimentos, partindo do pressuposto de Poor et al. (2013) de que a imagem de pessoas comendo produz efeitos na percepção de sabor pelo consumidor, qual a relação entre o tipo físico e possíveis diferenças no efeito produzido?

\section{A Influência do Meio de Apresentação}

No campo de marketing, muito já se estudou a respeito de como a presença de outras pessoas em um ambiente - e a imagem que elas representam para o consumidor - afetam o comportamento de ingestão de alimentos. Herman, Roth e Polivy (2003) tentaram compilar as diversas abordagens existentes em um quadro teórico visando à convergências destas. As escolhas relacionadas aos alimentos que um indivíduo consome diariamente são influenciadas por diversos elementos do contexto em que está inserido. Desse modo, entender como esses elementos afetam o processo de decisão é fator importante para qualquer profissional de marketing.

Diversos estudos têm investigado o papel que a influência social exerce no consumo alimentar (HERMAN; ROTH; POLIVY, 2003), principalmente como a quantidade consumida por determinado indivíduo é impactada pelas escolhas de outras pessoas.

As principais correntes no estudo do efeito da presença de outras pessoas nas escolhas de alimentos analisam: (a) o efeito do grupo nas quantidades consumidas; (b) como a quantidade consumida por outras pessoas afeta a escolha do consumidor; e (c) o efeito da sensação de estar sendo observado por outros na quantidade de ingestão de alimentos (HERMAN; ROTH; POLIVY, 2003). Entretanto a literatura não avança em outros constructos que, possivelmente, sofrem o efeito da presença de outras pessoas, como a percepção de sabor.

Ainda levando em conta o contexto de quantidade consumida, McFerran et al. (2010b) conduziram um estudo para avaliar o impacto do tipo físico de outras pessoas presentes e de suas escolhas na quantidade ingerida por parte do consumidor. Entretanto os autores evidenciam que a mera presença de outras pessoas, sem que haja menção ao consumo de alimentos, e seu tipo físico podem influenciar as escolhas (ex. uma garçonete). Contra intuitivamente, os autores evidenciam que a presença de uma garçonete com sobrepeso pode aumentar as quantidades consumidas de determinado produto. O presente artigo objetiva acessar a influência de outras pessoas presentes na percepção de sabor de alimentos não saudáveis em comparação com a imagem de pessoas vistas em anúncios impressos. O estudo, contudo, limita-se à investigação da influência de pessoas no ato de consumo do alimento, consoante trabalho anterior realizado por Poor et al. (2013).

Poor et al. (2013) sugerem que olhar imagens nas quais outros consumidores ingerem comidas não saudáveis diminui o conflito entre o afeto - relacionado ao sabor percebido do alimento - e a cognição 
- relacionada à preocupação com os efeitos do alimento para a saúde em longo prazo -, aumentando a percepção do sabor. O presente estudo, desse modo, constitui-se, sob esse prisma, em uma ampliação da abordagem realizada pelos autores, acrescentando a investigação do efeito relacionado à presença de outras pessoas no mesmo ambiente consumindo alimentos não saudáveis para a percepção do sabor por parte do consumidor.

Desse modo, com base nos conceitos revisados, o presente estudo tem por objetivo investigar as seguintes suposições:

H1 - A presença física de outra pessoa ingerindo o alimento tem mais impacto positivo na percepção de sabor pelo consumidor quando comparada à exposição a imagens de pessoas comendo o mesmo alimento

H2 - O tipo físico magro (acima do peso) do endossante resulta em uma percepção de sabor maior (menor) quando comparada com tipos físicos acima do peso (magro).

H3 - O gênero, moderado pelo tipo físico do endossante, influencia o sabor percebido pelo consumidor.

H3a - Para o tipo físico magro, não há diferença na percepção de sabor entre os gêneros masculino e feminino.

H3b - Para o tipo físico acima do peso, endossantes do gênero masculino (feminino) resultam em percepção de sabor maior (menor) quando comparados a endossantes do gênero feminino (masculino).

\section{Experimento 1}

O experimento foi desenvolvido com o objetivo de testar as hipóteses levantadas na sessão anterior. Desse modo, primeiramente, espera-se que os participantes submetidos à presença de outra pessoa consumindo fisicamente, no mesmo ambiente, um alimento não saudável, resulte em uma percepção de sabor maior do que os participantes submetidos a imagens de pessoas consumindo o mesmo alimento salgadinho do tipo batata chips. Além disso, espera-se que os indivíduos submetidos ao estímulo por meio de endossantes do tipo físico magro apresentem percepção de sabor superior aos do tipo físico acima do peso. Quanto ao gênero, a expectativa é de que a simples exposição a homens ou mulheres não altere a percepção de sabor; já a interação com o tipo físico deve apresentar resultados significativos, sendo que homens (mulheres) acima do peso ampliam (reduzem) o sabor percebido.

O alimento utilizado no estudo foi escolhido a partir de pré-teste realizado com estudantes de mesmo perfil amostral utilizado no experimento. O pré-teste foi aplicado com 78 estudantes de graduação, por meio de uma survey impressa, na qual os respondentes foram solicitados a classificar o quanto consideram saudável uma série de alimentos, em uma escala de 7 pontos ( $1=$ completamente saudável; $7=$ completamente não saudável). Dentre os alimentos percebidos como não saudáveis, o salgadinho do tipo batata chips foi o escolhido, em função de ter obtido a maior média $(M=6,14, S D=1,09)$, bem como pelo fato de que o alimento não poderia ter odor ressaltado, controlando uma possível fonte de problemas posteriores. Além disso, esse mesmo alimento foi também o utilizado por Poor et al. (2013) em sua pesquisa sobre percepção de sabor de alimentos não saudáveis.

\section{Método}

Participantes. No experimento, participaram cento e oitenta estudantes de graduação $(\mathrm{N}=180$, $57,8 \%$ Feminino, $M_{\text {idade }}=23,6$ ), que completaram o estudo em grupos de dez a vinte indivíduos, em uma sala previamente organizada para a realização da ação. A participação no estudo foi voluntária, no período entre aulas dos estudantes e sem qualquer tipo de compensação. Todos os participantes foram previamente informados de que estavam participando de um estudo sobre comportamento do consumidor e, antes do início da aplicação do experimento, foi esclarecido que aqueles que não concordassem em participar poderiam deixar a sala naquele momento. Cada indivíduo foi submetido a apenas uma condição experimental.

Manipulação. Foi empregado um design between-subjects factorial no formato 2 (forma de apresentação do estímulo: imagem ou presencial) x 2 (tipo físico do endossante: magro ou acima do peso) x 2 (gênero do endossante: feminino ou masculino). A metade dos participantes foi submetida a imagens impressas de pessoas consumindo salgadinho tipo batata chips, enquanto a outra metade teve a presença de mediadores 
consumindo o alimento no local de realização do experimento. As imagens e os mediadores foram ainda subdivididos em homens e mulheres, dos tipos físicos magro e acima do peso.

Procedimento. Todos os participantes, primeiramente, foram solicitados a responder algumas questões relativas à checagem dos elementos que podem influenciar os resultados. Primeiramente, os participantes deveriam responder, em uma escala de 7 pontos, seu nível de fome no momento de aplicação dos questionários (1 = sem fome; 7 = com muita fome). Em seguida, usando a mesma escala, perguntou-se o quanto gostam de vários alimentos, sendo um deles o salgadinho do tipo batata chips. Ao final da primeira etapa, foram solicitados o gênero e a idade do respondente.

Em seguida, foi realizada a manipulação do estudo. Solicitou-se que metade dos participantes avaliassem cinco imagens de pessoas consumindo o alimento (de acordo com o seu grupo experimental, que podia variar entre homens magros, homens acima do peso, mulheres magras e mulheres acima do peso). As imagens escolhidas foram de pessoas no ato consumatório, ou seja, com o alimento na boca, conforme procedimento realizado anteriormente por Poor et al. (2013). Após visualizar cada imagem impressa, os participantes avaliaram, em uma escala de 7 pontos, a atratividade da imagem (sendo 1 = "não me apetece" nada e 7 = "me apetece muito"). Os participantes submetidos à presença física de uma pessoa consumindo o alimento (nos mesmos subgrupos relacionados às imagens) foram solicitados a completar um jogo de caça-palavras. A cada intervalo de trinta segundos, o moderador solicitava a todos que prestassem atenção nele e dava uma instrução referente à atividade. Nesse momento, dava uma mordida no alimento escolhido, enquanto todos o assistiam. Esse procedimento foi repetido por cinco vezes.

Ao final da manipulação, foi informado aos participantes que havia um novo alimento enviado para teste. Assim, todos foram convidados a degustar uma pequena porção do alimento, sendo o intuito declarado da degustação realizar uma avaliação do alimento. Em seguida, foram solicitados a preencher cinco questões relacionadas à percepção de sabor do alimento, formatadas em escala de 7 pontos, as quais perguntavam sobre: o destaque do sabor ( 1 = "sem sabor destacado"; 7 = "com sabor destacado"); o quanto considera o alimento delicioso ( 1 = "pouco delicioso"; 7 = "muito delicioso"); a avaliação geral de sabor (1 = "pouco saboroso"; 7 = "muito saboroso"); e mais duas questões para mascarar o real intuito da avaliação, que perguntavam sobre a crocância e o preço em relação aos concorrentes.

Para finalizar, os participantes responderam a uma última série de perguntas, que consistiu na checagem das manipulações realizadas - se o participante percebeu o gênero e o tipo físico do endossante no estímulo a que foi submetido, além do alimento constante no estímulo. Foi solicitado, ainda, que os participantes respondessem a percepção sobre o quanto consideram o alimento consumido saudável/não saudável. Ao final do questionário foram feitas perguntas utilizadas para controle dos resultados, como a preocupação com um estilo de vida saudável por parte dos respondentes, sua percepção em relação ao próprio peso (se precisa perder ou ganhar peso), situação afetiva, peso e altura (para cálculo do IMC).

Ao final, foi realizado um breve debriefing, no qual ficou evidenciado que nenhum participante declarou conhecer as reais intenções do estudo. Aos participantes que solicitaram formalmente, ao final do experimento, foi encaminhado um breve sumário das reais intenções da pesquisa após o término da coleta de dados.

\section{Resultados}

A análise dos resultados foi realizada por meio de Análise de Covariância (ANCOVA), conforme as manipulações realizadas. Nos procedimentos iniciais de verificação da base de dados, foram excluídos nove casos que não passaram na checagem das manipulações realizadas - sete não souberam responder a qual alimento havia sido exposto e outros dois, ao gênero do endossante. Excluídos os nove casos, todos os participantes souberam responder qual o gênero de modelo e a quais alimentos foram submetidos.

A escala utilizada por Poor et al. (2013) para a percepção de sabor, composta dos itens já elencados anteriormente, foi testada e validada $(\alpha=.877)$. Desse modo, os itens foram agrupados em uma variável apenas, com valor correspondente à média aritmética. A análise por meio do score da regressão do primeiro fator extraído pela análise de componentes principais traz resultado semelhante. Com base na variável criada para avaliar a percepção de sabor, foram excluídos dois casos adicionais, os quais apresentam padrão de 
comportamento distante dos demais, considerados outliers. Desse modo, a análise realizada contou com 169 casos.

Os testes realizados indicam que os participantes, tal como no pré-teste realizado, consideraram o alimento a que foram expostos como não saudável $(M=6,18, S D=0,972)$. Em relação ao tipo físico dos modelos utilizados para apresentação dos estímulos, a checagem das manipulações indica que os modelos magros $(M=2,08, p<0,001)$ foram percebidos como mais magros que os modelos acima do peso $(M=$ 6,47 ) - as diferenças são significativas também quando isolados dentro de cada subgrupo (ex: imagens de pessoas do gênero feminino e presença de pessoas do gênero masculino).

As interações relacionadas ao meio de apresentação da imagem, conforme esperado, não apresentaram significância estatística $\left[F_{\text {meioxtipo fisico }}(1,159)=0,001, p=0,979 ; F_{\text {meioxgênero }}(1,159)=0,002, p=0,966\right.$; e $F_{\text {meioxtipo }}$ fisicoxgênero $(1,159)=0,515, p=0,474]$. Os resultados obtidos foram controlados pelo quanto os participantes gostam de batata $[F(1,159)=25,543, p<0,001)$ e por suas idades $(F(1,159)=5,211, p<0,05]$. Passamos, a seguir, à análise das hipóteses desta pesquisa.

Meio de apresentação do estímulo. Os resultados revelaram efeitos principais não significantes para o meio de apresentação do estímulo $[F(1,159=0,742, p>0,05]$, indicando a ausência de impacto da forma como o consumidor é submetido ao estímulo de outra pessoa ingerindo o alimento não saudável na percepção de sabor - se por meio de imagens ou da presença, fisicamente no mesmo ambiente, de alguém consumindo o alimento. Esse resultado é contrário ao esperado e rejeita a primeira hipótese do estudo, ressaltando a hipótese de Poor et al. (2013) de que a ampliação na percepção de sabor tem sua explicação na indulgência ao consumo provocada pelo estímulo de alguém consumindo o alimento - $\mathrm{e}$ independentemente do meio como esse estímulo é apresentado.

Tipo físico do endossante. Conforme o esperado, foi encontrada diferença significativa na percepção de sabor a partir do tipo físico dos endossantes $[F(1,159)=7,633, p<0,01]$. Especificamente, de acordo com a segunda hipótese do estudo, observa-se que, depois de controlado pelo quanto os indivíduos gostam de batata e pela idade dos respondentes, os endossantes do tipo físico magro $\left(\mathrm{M}_{\text {magro }}=5,450, \mathrm{p}<\right.$ 0,01 ) resultam em uma percepção de sabor superior do alimento não saudável quando comparados com os endossantes do tipo físico acima do peso $\left(\mathrm{M}_{\text {acima do peso }}=5,002\right)$. O mesmo padrão é repetido quando isolamos os resultados apenas entre os submetidos a imagens $\left(M_{\text {magro }}=5,53 ; M_{\text {acima do peso }}=5,05, p<0,05\right)$ ou à presença física do endossante $\left(M_{\text {magro }}=5,43 ; M_{\text {acima do peso }}=4,89, p=0,01\right)$, conferindo maior validade aos resultados. Assim, a segunda hipótese ficou comprovada pelo presente estudo, indicando que a percepção de sabor é influenciada pelo tipo físico do endossante de um anúncio, por exemplo. Tal contribuição é um avanço nos estudos de Poor et al. (2013), além de constituir uma contribuição para a teoria da propaganda.

Gênero do endossante. O gênero do modelo apresentado ao consumidor, de maneira isolada, conforme previsto no presente estudo, não produz efeito na percepção de sabor dos indivíduos $[F(1,159)$ $=0,17, p=0,898]$. Já a interação com o tipo físico do endossante, conforme predito, apresenta um impacto marginalmente significativo na variável dependente $[F(1,159)=2,658 p<0,1]$, indicando um suporte parcial à terceira hipótese do estudo. Esse resultado, embora marginalmente significante, representa um avanço significativo à literatura relacionada à percepção de sabor. $O$ efeito encontrado, entretanto, é diferente do previsto. Para os endossantes femininos, não há diferença significativa entre as percepções de sabor $\left(M_{\text {femXmagro }}=5,307, S D=0,158 M_{\text {femXacima do peso }}=5,123 \mathrm{SD}=0,167, p>0,05\right)$; já entre os modelos do gênero masculino, a exposição a endossantes magros traz uma percepção de sabor superior $\left(\mathrm{M}_{\text {mascxmagro }}=5,592\right.$ $\mathrm{SD}=0,161, \mathrm{p}<0,05)$ aos modelos acima do peso $\left(\mathrm{M}_{\text {mascXacima do peso }}=4,880 \mathrm{SD} 0,160\right)$. O efeito encontrado é apresentado graficamente abaixo. 


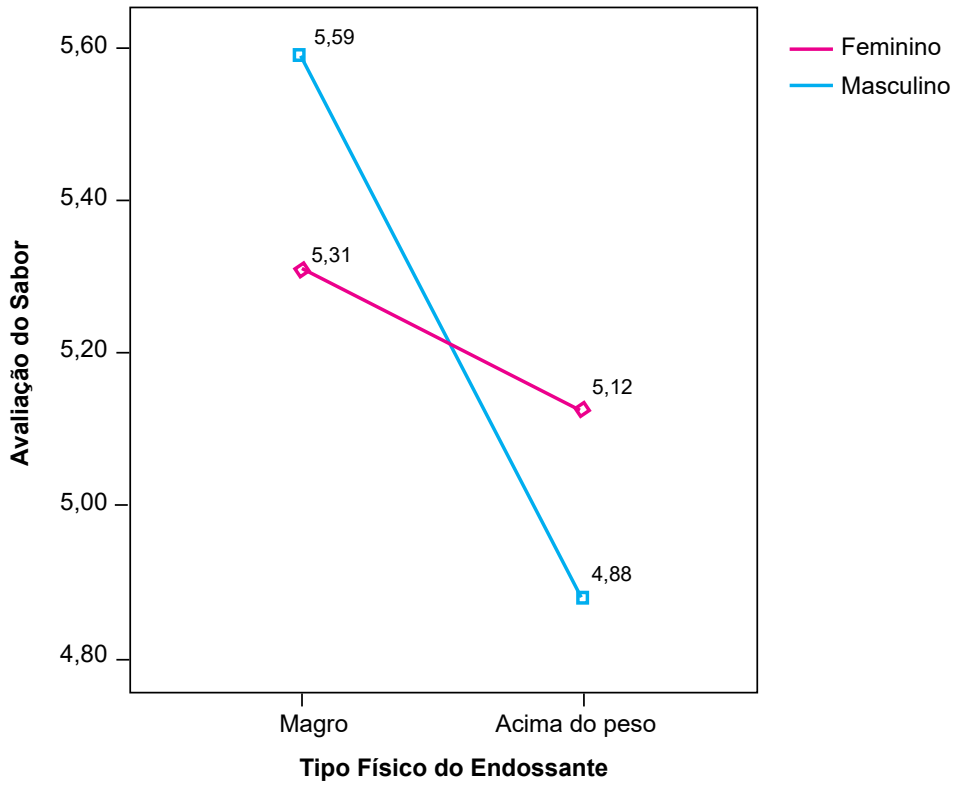

Fig 1: O efeito da interação do tipo físico e gênero do endossante na percepção de sabor pelo consumidor.

Percebe-se, a partir da leitura da figura, que existe uma sensibilidade maior relacionada à percepção de sabor quando da utilização de modelos masculinos, sendo que os acima do peso causam impacto negativo na avaliação do alimento não saudável. Um estudo posterior, visando à ampliação da investigação desse efeito de interação, se faz necessário, de modo a comprovar/rejeitar o efeito marginalmente identificado.

\section{Discussão}

O presente artigo buscou ampliar as descobertas de Poor et al. (2013), que evidenciaram que, para alimentos não saudáveis, a utilização de imagens consumatórias - de alguém comendo o alimento aciona nos indivíduos um objetivo de indulgência, reduzindo os conflitos psicológicos relacionados à saúde resultantes do consumo. Como consequência, o consumidor avalia de forma superior esses alimentos e, frequentemente, mostra-se mais disposto a escolher alimentos não saudáveis.

Primeiramente, o estudo, ao rejeitar a primeira hipótese, proposta como pauta para futuras investigações pelos autores - que tinha como pressuposto uma percepção de sabor superior quando há alguém consumindo fisicamente o alimento no mesmo ambiente comparado a imagens -, reforça a explicação psicológica relacionada ao acionamento de objetivos (Poor et al., 2013). Desse modo, mais importante do que o meio no qual o consumidor tem contato com o estímulo, o determinante para a avaliação realizada parece ser mesmo o fato de uma pessoa estar consumindo o alimento não saudável.

O tipo físico do endossante de anúncio já foi explorado em vários estudos na literatura de marketing como influencia as avaliações dos consumidores ou a atitude em relação à marca, por exemplo. Entretanto a avaliação positiva de uma peça publicitária, isoladamente, pode ser uma fonte de explicação insuficiente nas escolhas de consumo. Nesse cenário, a confirmação da segunda hipótese do estudo, confirmando que a utilização de modelos magros se traduz em uma avaliação mais positiva quando comparada com o tipo físico acima do peso, traz novos pressupostos a se observar quando da formulação de propagandas. Além disso, o fato de os padrões encontrados repetirem-se quando isolados os participantes que receberam o estímulo fisicamente evidencia a possibilidade de obter benefícios também a partir definição do leiaute das lojas. Do ponto de vista da literatura de marketing, contribui para uma ampliação do entendimento da percepção de sabor e, especificamente, do mecanismo psicológico que leva à indulgência no consumo de alimentos não saudáveis.

A terceira hipótese, marginalmente comprovada, é aquela que constitui a principal contribuição do presente estudo. Foi evidenciado que, para endossantes magros, não há diferença na percepção de sabor quando da utilização dos gêneros masculino ou feminino; já para modelos acima do peso, ao contrário do 
esperado, endossantes do gênero masculino parecem resultar em uma avaliação menos positiva por parte dos consumidores. Essa evidência, entretanto, traz a necessidade de um aprofundamento na investigação da hipótese, principalmente se considerarmos o efeito de moderação sugerido pelos resultados. A comprovação da hipótese consiste em um avanço ainda mais significativo nos pontos já discutidos em relação à segunda hipótese deste estudo, e significa um avanço importante na literatura sobre o tema, contribuindo de forma determinante para uma compreensão mais ampla dos efeitos psicológicos evidenciados por Poor et al. (2013) e do próprio estudo realizado. Além disso, entre as modelos do sexo feminino, avançando nas descobertas de Forbes et al., (2001), o presente estudo mostra que a percepção de sabor não é alterada quando da utilização de modelos acima do peso, o que pode significar novos conceitos a explorar na propaganda de alimentos não saudáveis.

\section{Referências}

Ariely, D., \& Levav, J. (2000). Sequential choice in group settings: Taking the road less traveled and less enjoyed. Journal of consumer Research, 27(3), 279-290.

Bjerke, R., \& Polegato, R. (2006). How well do advertising images of health and beauty travel across cultures? A self-concept perspective. Psychology \& Marketing, 23(10), 865-884.

Chernev, A. (2011). The dieter's paradox. Journal of Consumer Psychology, 21(2), 178-183.

Chandon, P., \& Wansink, B. (2012). Does food marketing need to make us fat? A review and solutions. Nutrition reviews, 70(10), 571-593.

Chowdhury, R. M., Olsen, G. D., \& Pracejus, J. W. (2008). Affective responses to images in print advertising: Affect integration in a simultaneous presentation context. Journal of Advertising, 37(3), 7-18.

D'alessandro, S., \& Chitty, B. (2011). Real or relevant beauty? Body shape and endorser effects on brand attitude and body image. Psychology \& Marketing, 28(8), 843-878.

Dattatreya, B. S., Kamath, A., \& Bhat, K. K. (2002). Developments and challenges in flavor perception and measurement-a review. Food Reviews International, 18(2-3), 223-241.

Elder, R. S., \& Krishna, A. (2010). The effects of advertising copy on sensory thoughts and perceived taste. Journal of consumer research, 36(5), 748-756.

Forbes, G. B., Adams-Curtis, L. E., Rade, B., \& Jaberg, P. (2001). Body dissatisfaction in women and men: The role of gender-typing and self-esteem. Sex Roles, 44(7-8), 461-484.

Ghemulet, M., Baskini, M., Messinis, L., Mouza, E., \& Proios, H. (2014). Taste perception analysis using a semantic verbal fluency task. Psychology Research and Behavior Management, 7, Número?, 261-272.

Givon, M. M., \& Goldman, A. (1987). Perceptual and preferential discrimination abilities in taste tests. Journal of applied psychology, 72(2), 301.

Guichard, E. (2002). Interactions between flavor compounds and food ingredients and theirinfluence on flavor perception. Food Reviews International, 18(1), 49-70.

Herman, C. P., Roth, D. A., \& Polivy, J. (2003). Effects of the presence of others on food intake: a normative interpretation. Psychological bulletin, 129(6), 873.

Hong, J. W., \& Zinkhan, G. M. (1995). Self-concept and advertising effectiveness: The influence of congruency, conspicuousness, and response mode. Psychology \& Marketing, 12(1), 53-77.

Killgore, W. D., Young, A. D., Femia, L. A., Bogorodzki, P., Rogowska, J., \& Yurgelun-Todd, D. A. (2003). Cortical and limbic activation during viewing of high-versus low-calorie foods. Neuroimage, 19(4), 13811394.

Levy, S. J. (1959). Symbols By Which We Buy. In L. H. Stockman (Ed.), Advancing Marketing Efficiency (pp. 409-416). Chicago: American Marketing Association. 
Liem, D. G., Aydin, N. T., \& Zandstra, E. H. (2012). Effects of health labels on expected and actual taste perception of soup. Food quality and preference, 25(2), 192-197.

Lynch, J. G., Jr., Ariely, D. (1998). Wine online: Search cost and competition on price, quality, and distribution. Marketing Science,Volume?, númeor?, página?.

Madzharov, A. V., \& Block, L. G. (2010). Effects of product unit image on consumption of snack foods. Journal of Consumer Psychology, 20(4), 398-409.

McClure, S. M., Botvinick, M. M., Yeung, N., Greene, J. D., \& Cohen, J. D. (2007). Conflict monitoring in cognition-emotion competition. Handbook of emotion regulation, Volume?, número?, 204-226.

McFerran, B., Dahl, D. W., Fitzsimons, G. J., \& Morales, A. C. (2010a). I'll have what she's having: Effects of social influence and body type on the food choices of others. Journal of Consumer Research, 36(6), 915-929.

McFerran, B., Dahl, D. W., Fitzsimons, G. J., \& Morales, A. C. (2010b). Might an overweight waitress make you eat more? How the body type of others is sufficient to alter our food consumption. Journal of Consumer Psychology, 20(2), 146-151.

Mishra, A., \& Mishra, H. (2011). The influence of price discount versus bonus pack on the preference for virtue and vice foods. Journal of Marketing Research, 48(1), 196-206.

Mizutani, N., Okamoto, M., Yamaguchi, Y., Kusakabe, Y., Dan, I., \& Yamanaka, T. (2010). Package images modulate flavor perception for orange juice. Food quality and preference, 21(7), 867-872.

Mizutani, N., Dan, I., Kyutoku, Y., Tsuzuki, D., Clowney, L., Kusakabe, Y., ... \& Yamanaka, T. (2012). Package images modulate flavors in memory: Incidental learning of fruit juice flavors. Food quality and preference, 24(1), 92-98.

Nowlis, S. M., \& Shiv, B. (2005). The influence of consumer distractions on the effectiveness of foodsampling programs. Journal of Marketing Research, 42(2), 157-168.

Ohla, K., Le Coutre, J., \& Hudry, J. (2010). How what we see affects what we taste. Perception ECVP abstract, 39 Número ?, 145-145.

Ohla, K., Toepel, U., Le Coutre, J., \& Hudry, J. (2012). Visual-gustatory interaction: orbitofrontal and insular cortices mediate the effect of high-calorie visual food cues on taste pleasantness. PloS one, $7(3)$ página? .

Okada, E. M. (2005). Justification effects on consumer choice of hedonic and utilitarian goods. Journal of marketing research, 42(1), 43-53.

Poor, M., Duhachek, A., \& Krishnan, H. S. (2013). How images of other consumers influence subsequent taste perceptions. Journal of Marketing, 77(6), 124-139.

Read, D., Loewenstein, G., \& Kalyanaraman, S. (1999). Mixing virtue and vice: Combining the immediacy effect and the diversification heuristic. Journal of Behavioral Decision Making, 12(4), 257.

Rosenberg, M. (1979). Conceiving the Self. Malabar, FL: Robert e Krieger.

Shiv, B., \& Nowlis, S. M. (2004). The effect of distractions while tasting a food sample: The interplay of informational and affective components in subsequent choice. Journal of Consumer Research, 31(3), 599-608.

Small, D. M. (2012). Flavor is in the brain. Physiology \& behavior, 107(4), 540-552.

Smeesters, D., Mussweiler, T., \& Mandel, N. (2010). The effects of thin and heavy media images on overweight and underweight consumers: Social comparison processes and behavioral implications. Journal of Consumer Research, 36(6), 930-949.

Solomon, M. R., \& Douglas, S. P. (1987). Diversity in product symbolism: The case of female executive clothing. Psychology \& Marketing, 4(3), 189-212. 
Underwood, R. L., \& Klein, N. M. (2002). Packaging as brand communication: effects ofproduct pictures on consumer responses to the package and brand. Journal of Marketing Theory and Practice, 10(4), 5868.

Yang, X., Zhang, J., \& Peracchio, L. A. (2010). Understanding the impact of self-concept on the stylistic properties of images. Journal of Consumer Psychology, 20(4), 508-520.

Submetido em: $26 / 06 / 2018$

Aceito em: 10/04/2019 